\section{MFA haben Anspruch auf Weihnachtsgeld}

\section{Nach dem Tarifvertrag für medizinische Fachangestellte ist das 13. Monatsge- halt keine freiwillige Leistung, sondern Pflichtübung für Praxischefs.}

U rsprünglich ist das Weihnachtsgeld eine freiwillige Leistung des Arbeitgebers, mit der Betriebstreue und Arbeitsleistung honoriert werden können. Wird es in Betriebsvereinbarungen, Arbeits- oder Tarifverträgen zugesagt, dürfen Arbeitgeber die Zahlung aber nicht einfach einstellen.

\section{Knackpunkt für Praxischefs}

Nach dem aktuell gültigen Manteltarifvertrag für medizinische Fachangestellte (MFA) steht diesen ein 13. Monatsgehalt $\mathrm{zu}$, das spätestens bis zum ersten Dezember an die Fachangestellten auszuzahlen ist. Dabei beträgt es mindestens die Höhe des letzten vollen Monatsgehalts (Paragraf 12 Manteltarifvertrag).

Wichtig ist: Der Tarifvertrag gilt nicht nur für Praxen, die Mitglied in der Tarifpartei auf Arbeitgeberseite - also der
Arbeitsgemeinschaft zur Regelung der Arbeitsbedingungen von MFA/Arzthelferinnen (AAA) - sind. Es reicht aus,

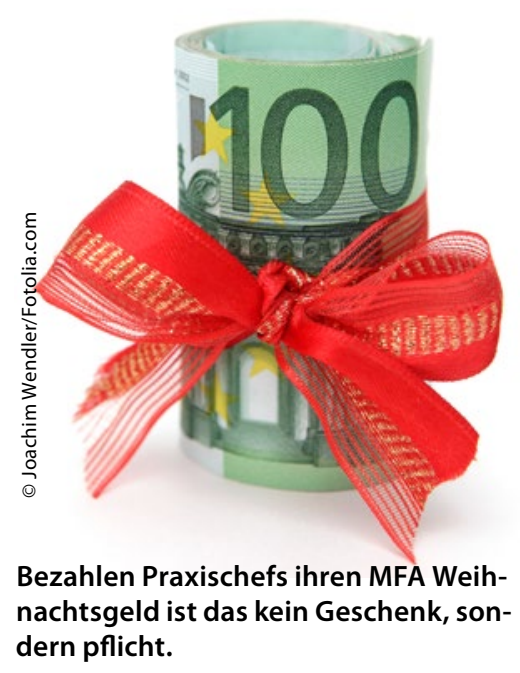
dern pflicht. wenn sich der Arbeitsvertrag der MFA auf den geltenden Mantel- und Gehaltstarif bezieht. Insbesondere die Musterarbeitsverträge der Bundesärztekammer und des Verbands medizinischer Fachberufe $(\mathrm{VmF})$ enthalten einen entsprechenden Passus.

Selbst wenn der Bezug zum Tarifvertrag nicht gegeben ist: Wurde mehrere Jahre hintereinander Weihnachtsgeld gezahlt, ohne dass der Arbeitgeber ausdrücklich jedes Jahr neu die Freiwilligkeit betont hat, besteht ebenfalls ein Anspruch auf Zahlung. Nach der gängigen Rechtsprechung liegt dann nämlich ein Fall von betrieblicher Übung vor - und meist reichen dafür schon drei aufeinanderfolgende Jahre aus.

In diesem Jahr bekommen übrigens gut die Hälfte (54 \%) der deutschen Beschäftigten Weihnachtsgeld $-56 \%$ in West- und $40 \%$ in Ostdeutschland. Das hat das Tarifarchiv der gewerkschaftsnahen Hans-Böckler-Stiftung in einer Online-Umfrage mit 8.800 Beteiligten herausgefunden. Grundlage sind dabei meist Tarifverträge.

Rebecca Höhl/dpa

Neue GOÄ

\title{
Gebührenrahmen soll stark eingeschränkt werden
}

Das geplante Paragrafenwerk der neuen GOÄ sieht einige massive Änderungen vor. So soll es auch bei der Abrechnung von Leistungen außerhalb des Fachgebietes Einschränkungen geben.

D ie Steigerungsmöglichkeiten bei Gebühren für Leistungen nach der neuen GOÄ sollen erheblich eingeschränkt werden: Der Entwurf des Paragrafenwerks der neuen Gebührenordnung sieht unter anderem vor, dass eine Steigerung des einfachen Gebührensatzes nur noch unter vorher von einer ,gemeinsamen Kommission" festgelegten Umständen „aufgrund der objektiven Schwere des Einzelfalls" und nur noch um den Faktor 2 möglich sein soll.

Der Entwurf, der von der Bundesärztekammer zusammen mit dem Verband der Privaten Krankenversicherung und Vertretern der Beihilfe erarbeitet worden ist, liegt dem Springer Medizin Verlag vor. Aus früheren Ankündigungen der Bundesärztekammer zur neuen GOÄ geht hervor, dass die Einschränkung beim Gebührenrahmen dadurch möglich werden soll, dass ein „robuster Einfachsatz" bereits so kalkuliert ist, dass in sehr vielen Fällen eine Steigerung nicht mehr nötig sein wird. Die Legenden und Bewertungen der einzelnen Leistungen werden bisher allerdings noch unter Verschluss gehalten. Nach den Plänen der Initiatoren der neuen GOÄ wird das Gebührenwerk im Oktober 2016 in Kraft treten, also noch vor Beginn des Bundestagswahlkampfes.
Anders als bisher steht der Punktwert für die Leistungen nicht mehr im Paragrafenteil der GOÄ, sondern wird erst später bekannt gegeben, wenn die Bewertungen der Leistungen zwischen den Verhandlungspartnern konsentiert sind.

\section{Weitere stärkere Reglementierung}

Auch an anderen Stellen steht eine stärkere Reglementierung bevor. So heißt es, dass der Arzt nur Vergütungen für Leistungen berechnen darf, „für deren Erbringung der Arzt nach Maßgabe des Weiterbildungsrechts grundsätzlich die fachliche Qualifikation besitzt". Die geäußerte Kritik des Spitzenverbandes der Fachärzte (SpiFa) und der Zahnärzte richtet sich zum einen gegen die zunehmende Reglementierung, zum anderen gegen „Lösungen zur modellhaften Erprobung von Elementen zur Verbesserung der Versorgungsstruktur", die in dem Gebührenwerk ebenfalls vorgesehen sind. Die Verbände sehen darin die Einführung von Öffnungsklauseln für die GOÄ durch die Hintertür. Hauke Gerlof 